\title{
Experimental Generation and Observation of Intrinsic Localized Spin Wave Modes in an Antiferromagnet
}

\author{
U. T. Schwarz, L. Q. English, and A. J. Sievers \\ Laboratory of Atomic and Solid State Physics, Cornell University, Ithaca, New York 14853-2501
}

(Received 9 April 1999)

\begin{abstract}
By driving with a microwave pulse the lowest frequency antiferromagnetic resonance of the quasi-1D biaxial antiferromagnet $\left(\mathrm{C}_{2} \mathrm{H}_{5} \mathrm{NH}_{3}\right)_{2} \mathrm{CuCl}_{4}$ into an unstable region, intrinsic localized spin waves have been generated and detected in the spin wave gap. These findings are consistent with the prediction that nonlinearity plus lattice discreteness can lead to localized excitations with dimensions comparable to the lattice constant.
\end{abstract}

PACS numbers: 76.50.+g, 05.45.-a, 75.50.Ee

Although solitons continue to play an important role in condensed matter physics [1-3] in the last decade it was recognized that nonlinearity plus lattice discreteness can lead to a different class of excitations with dimensions comparable to the lattice constant [4-6]. Such intrinsic localized modes (ILMs) have been identified in a variety of classical molecular dynamics (MD) simulations [7-9] and with macroscopic mechanical [10] and electrical [11] models, all of which ignore the possible role of quantum mechanics [12]. Some effort has gone into identifying specific condensed matter signatures as evidence of ILM production but all require intricate arguments: these include far infrared absorption [13], radiation ionization tracks [14], the temperature dependent Mössbauer effect [4], and resonant Raman scattering [15]. As yet evidence of externally generated ILMs in a lattice of atomic dimensions is missing. The large amplitude modulational instability of an antiferromagnetic resonance (AFMR) for some antiferromagnets has been suggested [16,17] as a mechanism for the generation of intrinsic localized spin wave modes (ILSMs) and in this Letter we describe the experimental observation and control of such nanoscale excitations.

Because the driving field necessary to create ILMs via the modulational instability scales with the frequency of the antiferromagnetic resonance, $\omega_{\mathrm{AFMR}}$, $\left(\mathrm{C}_{2} \mathrm{H}_{5} \mathrm{NH}_{3}\right)_{2} \mathrm{CuCl}_{4}$ with the lowest frequency resonance at $\omega_{\mathrm{AFMR}}=1.5 \mathrm{GHz}$ was chosen for this first study. This antiferromagnet, often referred to as $\mathrm{C}(2) \mathrm{CuCl}_{4}$, is a layered organic material with a strong ferromagnetic coupling of the magnetic $\mathrm{Cu}^{2+}$ ions within a layer and with a weak antiferromagnetic coupling between these layers. Because of this weak interlayer coupling the total spin in each layer can be represented by a classical one with respect to describing the lowest frequency mode dynamics. This spin system with its biaxial anisotropy is described in more detail in Ref. [17].

The $\mathrm{C}(2) \mathrm{CuCl}_{4}$ single crystals were grown from aqueous solution of ethylamine hydrochloride and copper (II) chloride in a closed vessel by slowly decreasing the temperature [18]. For the measurement, platelets with well defined surfaces and a typical dimension of $3 \times 3 \times$ $0.5 \mathrm{~mm}^{3}$ were chosen.

The experimental setup is shown schematically in Fig. 1(a). The first oscillator provides the high power pulse and the second oscillator the probe beam. Because the expected experimental signature of ILSM generation after a strong microwave pulse near the $\omega_{\text {AFMR }}$ is the breakup of the AFMR into a broad band, the generation/detection system is tailored to produce a large ac field at the driving frequency $\omega_{\text {excite }}$ and a high sensitivity over a broad band below but close to $\omega_{\text {excite. }}$ A

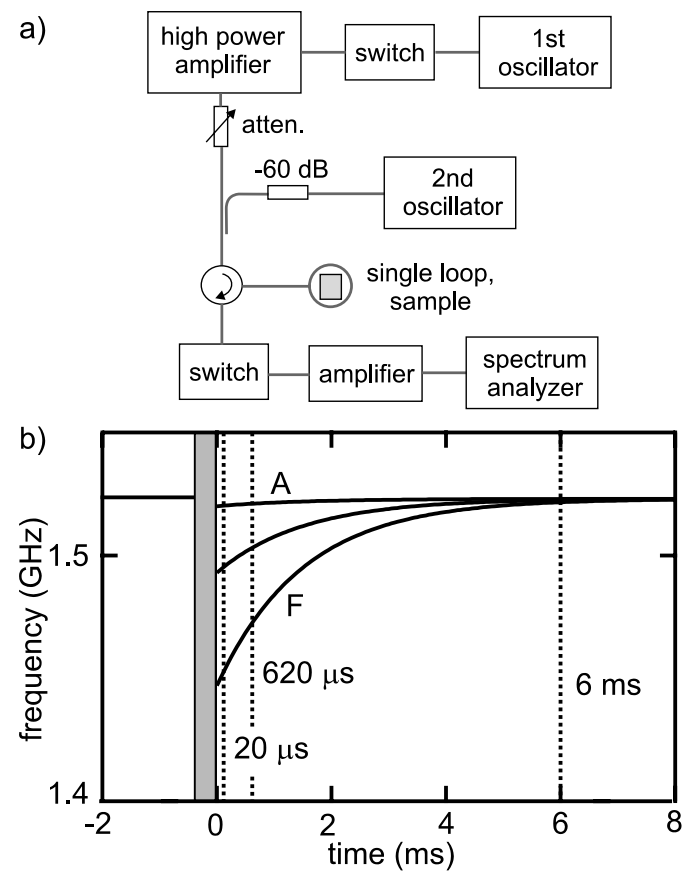

FIG. 1. (a) Schematic experimental setup. Details are given in the text. (b) Experimental measurement road map. The horizontal line for $t<0$ identifies the AFMR frequency. During the high power driving pulse (shaded area) the detection electronic is blocked. The solid lines $A-F$ show the AFMR for increasing pump-pulse power levels. The vertical dotted lines identify different time cuts described in the text. 
single $3 \mathrm{~mm}$ diameter loop of copper wire is used as a nonresonant antenna for the excitation and pickup of the broad band signal in reflection. A pump signal of up to $100 \mathrm{~W}$ can be obtained from the oscillator and solid state amplifier. To create short pulses a fast GaAs switch is used in front of the amplifier. The signal of a second, tunable oscillator is overlayed by means of a directional coupler. With a second fast switch, synchronized by a digital delay generator, the reflected signal from the pump pulse is suppressed. The weak reflected signal from the second oscillator passes through two low noise amplifiers and is detected with a spectrum analyzer which was used as a variable bandwidth detector locked to the frequency of the probe oscillator. The minimum noise level of the system is $-120 \mathrm{dBm}$ at $100 \mathrm{kHz}$ bandwidth. In this measurement setup the absorption can be obtained as a function of time and frequency by first measuring the time dependent absorption at the frequency of the second oscillator, and then scanning this oscillator frequency during subsequent pump pulses. The time resolution is limited to $20 \mu$ s by the spectrum analyzer, and the sensitivity, by the flatness of the exciting loop impedance. Care was taken not to saturate any electronic circuits, as this could produce an artificial nonlinear response. The exciting coil and sample are immersed in pumped liquid helium at $1.2 \mathrm{~K}$.

To provide a road map to the experimental data-taking procedure a schematic view of the power and time dependence of the AFMR is shown in Fig. 1(b). The AFMR frequency before the pulse, $t<0$, is represented by the horizontal line. During the high power driving pulse (shaded area) the detection electronics is blocked. After the trailing edge $(t=0)$ of the driving pulse the spin wave is highly excited and its frequency is decreased due to the intrinsic nonlinearity of the spin Hamiltonian. From this state the system relaxes back to equilibrium with the longitudinal relaxation time $T_{1}$. The absorption by the spin system can be examined for different power levels of the driving pulse [lines labeled $A-F$ in Fig. 1(b)] or for different time delays at a fixed power [dotted lines in Fig. 1(b)] or as a function of the driving pulse length. Measurements varying all of these experimental parameters have been carried out and are described below.

Figure 2 shows the absorption spectra $20 \mu \mathrm{s}\left(\gg T_{2}\right.$ of the AFMR) after the trailing end of a pump pulse for six different powers. The power of the $400 \mu$ s long driving pulse varied from $50 \mathrm{~mW}$ to $1.6 \mathrm{~W}$. On the low frequency side of each spectrum are two magnetostatic volume modes while the shoulder on the high frequency side is a surface mode [19]. With increasing power levels the AFMR first collapses into a broad asymmetric shape and then at still higher powers returns to a sharp AFMR. Note that the magnetostatic modes do not show the same behavior as the AFMR. These experimental results demonstrate that the AFMR is indeed unstable with increasing amplitude but only up to a specific amplitude

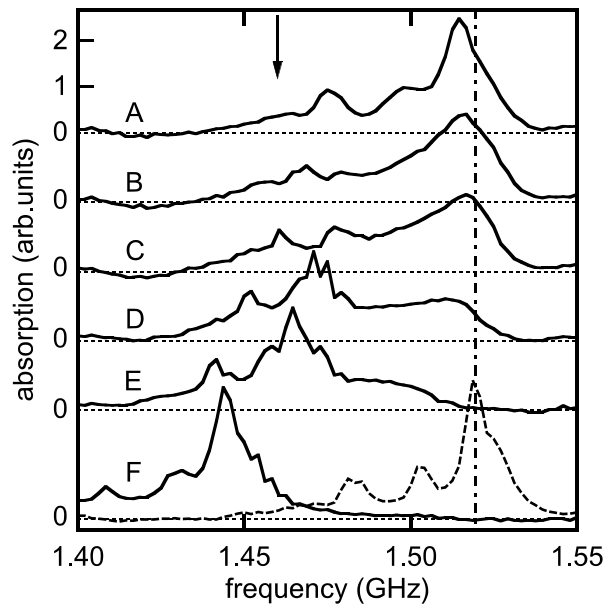

FIG. 2. Absorption spectra measured $20 \mu \mathrm{s}$ after a $400 \mu \mathrm{s}$ driving pulse for the different power levels; $(A) 50 \mathrm{~mW},(B)$ $100 \mathrm{~mW},(C) 160 \mathrm{~mW},(D) 320 \mathrm{~mW},(E) 500 \mathrm{~mW}$, and $(F)$ $1.6 \mathrm{~W}$ of the $1.46 \mathrm{GHz}$ (arrow) driving pulse. For clarity the spectra are shifted on the linear ordinate. The asymmetric wing on the low frequency side of the AFMR is the expected signature of localized mode production. The dot-dashed line marks the lowest frequency $\omega_{\mathrm{AFMR}}$ for an applied static field $H_{\mathrm{dc}}=12 \mathrm{mT}$. The dashed spectrum was taken at low power before the driving pulse.

while for still larger amplitudes the AFMR uniform mode again becomes stable.

The results in Fig. 2 illustrate some of the criteria for the creation of ILSMs. First there is a minimum transverse amplitude above which the extended mode becomes unstable but then, surprisingly, there is also a maximum amplitude associated with this instability of the extended mode. This was not expected from our molecular dynamics simulations. Next the frequency interval of the instability region is observed to become larger as the frequency shift $\omega_{\text {excite }}-\omega_{\mathrm{AFMR}}$ becomes larger. For optimum conditions, localization could be observed for powers as low as $50 \mathrm{~mW}$ in a $400 \mu$ s driving pulse. Varying the pulse width from 50 to $400 \mu$ s while keeping the energy in the pulse fixed does not change these criteria.

The narrow linewidth of curve $F$ shown in Fig. 2 after the strongest driving pulse is a handy test to exclude any temperature related effect, such as the heating of the crystal by the intense microwave pulses. From low power linear measurements both the temperature dependence of the AFMR frequency, which goes to zero at the Néel temperature $T_{N}=10.2 \mathrm{~K}$, and its linewidth are known. If the frequency shift of $75 \mathrm{MHz}$ between the low power trace (dashed line in Fig. 2) and high power trace were caused by an increased temperature in the crystal, the linewidth should nearly double. This is not observed so temperature effects are not the source of the unusual line shape results.

The frequency response at several delay times for $200 \mathrm{~mW}$ excitation in a $200 \mu$ s pulse is shown in Fig. 3. 


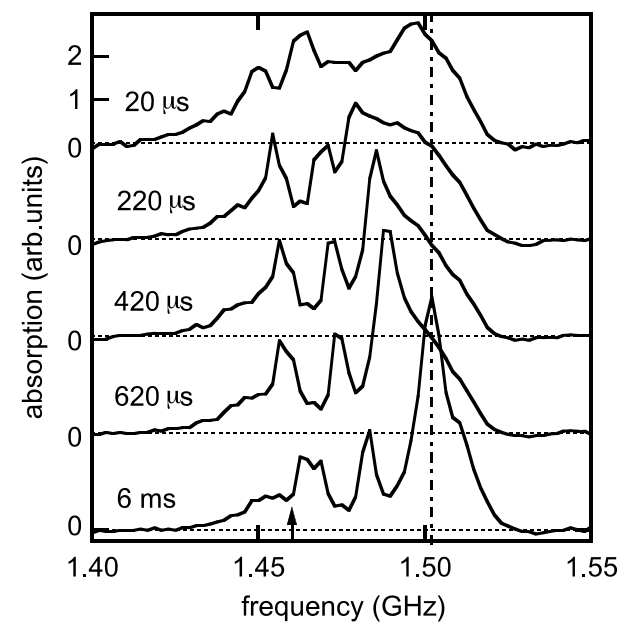

FIG. 3. Absorption spectra measured $20 \mu \mathrm{s}, 220 \mu \mathrm{s}, 420 \mu \mathrm{s}$, $620 \mu \mathrm{s}$, and $6 \mathrm{~ms}$ after a $200 \mu \mathrm{s}$ long driving pulse at $1.46 \mathrm{GHz}$ (arrow). The spectra are shifted on the ordinate by equal amounts. The dot-dashed line marks $\omega_{\text {AFMR }}$ as in Fig. 2.

The five traces at $20 \mu \mathrm{s}, 220 \mu \mathrm{s}, 420 \mu \mathrm{s}, 620 \mu \mathrm{s}$, and $6 \mathrm{~ms}$ show the evolution of this absorption feature from a broad resonance back to an AFMR at times smaller than $T_{1}=1.50 \mathrm{~ms}$. For times larger than $2 T_{1}$ only the AFMR survives. The radical difference between the line shapes at $t=20 \mu \mathrm{s}$ and $t=420 \mu \mathrm{s}$ is consistent with the idea that the breakup into ILSMs is possible only when the effects of nonlinearity and dispersion are much stronger than the dissipation effect [16].

MD simulations can be used to demonstrate that the extended spin wave is unstable against breakup into localized modes for the specific amplitude of the extended wave produced in our experiments. Details on molecular dynamics procedures for simulating localized spin waves in $\mathrm{C}(2) \mathrm{CuCl}_{4}$ can be found in Ref. [17]. The solid line in Fig. 4(a) shows the calculated frequency dependence of the AFMR as a function of its transverse amplitude $S_{y}$ in the hard axis direction. To compare this simulation to our experiment, we extract from the shift of $75 \mathrm{MHz}$ or $0.05 \omega_{\mathrm{AFMR}}$ of trace $(F)$ in Fig. 2 the spin wave amplitude $S_{y}=0.06$ as marked with $(F)$ in Fig. 4(a). Correspondingly the points $A$ to $F$ superimposed on this curve identify different AFMR frequency shifts observed in the high power measurements of Fig. 2. To test for the instability threshold the time dependent evolution of the energy density for a simulation of a 250 spin antiferromagnetic chain is calculated, starting from an extended wave with fixed transverse amplitude plus random noise $\left\langle\delta S_{n}\right\rangle=0.0025$. For the whole range $(A)$ to $(F)$ covered by the experiment the extended spin wave is unstable and breaks up into localized modes with widths extending over about ten lattice constants. Thus for these microwave powers the AFMR is driven into the interesting nonlinear region.

To compare the absorption spectrum $A(\omega) \propto \omega \chi^{\prime \prime}$ measured in the experiment with results from molecular
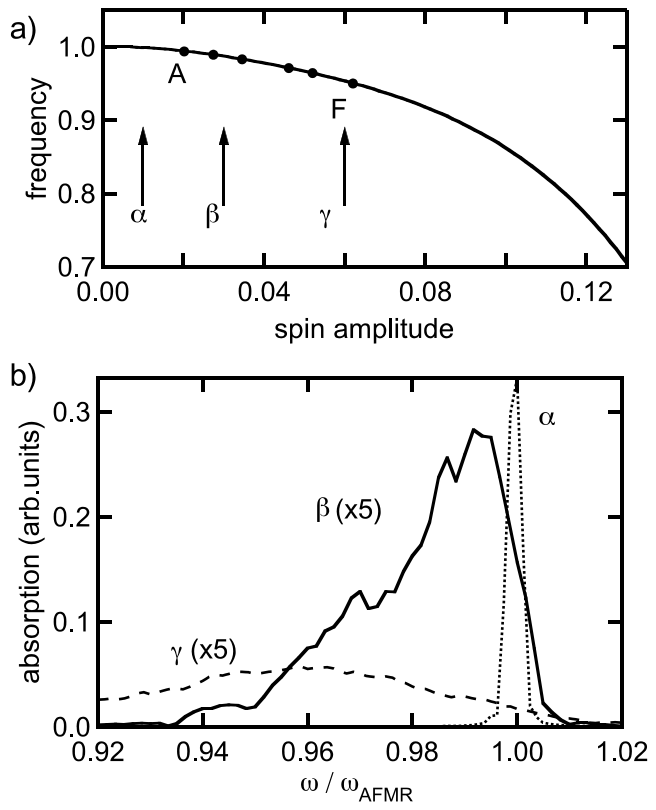

FIG. 4. (a) Calculated frequency of the uniform spin wave mode as function of its transverse amplitude. $(A)$ to $(F)$ mark the experimentally determined position of the extended mode after the driving pulse from the corresponding traces in Fig. 2. (b) AFMR plus ILSM absorption spectra determined from molecular dynamics simulations corresponding to three different microwave power levels. Starting from extended spin waves with a given transverse amplitude of $S y=0.01,0.03$, and 0.06 [arrow positions $\alpha, \beta$, and $\gamma$ in (a)] the uniform mode breaks up into ILSMs within a time of $200 \tau_{\mathrm{AFMR}}$. From the evolution over the subsequent time interval $\Delta \tau=800 \tau_{\mathrm{AFMR}}$ the spectra are obtained via the autocorrelation of the net transverse magnetic moment as described in the text.

dynamic simulations, the imaginary part of the dynamic magnetic susceptibility is calculated using the Kubo expression [20] where the absorption is proportional to the Fourier transform of the autocorrelation function of the net magnetic moment, $M_{y}(t)$ :

$$
\chi_{y}^{\prime \prime}(\omega) \propto \omega \int_{0}^{\infty} d t\left\langle M_{y}\left(t_{0}+t\right) M_{y}(t)\right\rangle \exp (i \omega t) .
$$

For our special case the simulations are started with an extended wave of a given amplitude plus some small amount of random noise. The extended wave is unstable against breakup into localized excitations and transforms after approximately 100 periods of the antiferromagnetic resonance into a broad spectrum of ILMs. From 200 to $1000 \tau_{\mathrm{AFMR}}$ the evolution of the net magnetic moment is recorded and then via Eq. (1) the imaginary part of the dynamic magnetic susceptibility is found.

The resulting calculated absorption spectra for a chain of 1000 spins at three different powers are shown in Fig. 4(b). To generate these spectra the starting transverse amplitudes of the AFMR are $S y=0.01,0.03$, and 0.06, respectively. Each curve is averaged over six simulation 
runs to remove arbitrary spikes associated with single longliving ILSMs. The broad wing of the asymmetric band is due to the statistical distribution of the ILMs with different degrees of localization and thus different frequencies. To compare the simulations on the long chain with the spectra obtained in the experiment, the frequency axis in Fig. 4(b) was scaled to the same width relative to $\omega_{\text {AFMR }}$ as given in Figs. 2 and 3. Both the shape and width of the experimental onset results are in good agreement with theoretical simulations. Moreover the absorption spectra shown in Fig. 4(b) are obtained starting from amplitudes of the extended wave which are in the right range for traces $A$ to $F$.

The disappearance of the instability at large amplitudes is an experimental feature not represented by curve $\gamma$ in these model calculations. Since the values of the relaxation times taken from the experiment are $T_{1}=$ $1.50 \mathrm{~ms}$ and $T_{2} \approx 0.1 \mu \mathrm{s}$, with the latter depending on the surface quality of the samples, $T_{2}$ is much smaller than the pulse widths used in our experiments so that energy is transferred to other degenerate spin waves during the microwave pulse. Our experimental results indicate that at the powers where the uniform mode instability occurs this transfer does not influence the positive curvature of the dispersion curve which is required for the instability. At the highest powers shown in Fig. 2 the uniform mode again becomes stable indicating that the finite wave vector spin waves have become so heavily populated during the microwave pulse that the dispersion curve now has negative curvature at the uniform mode, a condition for stability of the mode at large amplitude [16]. Because it takes a finite time for the uniform mode to break up into ILSMs both features can appear at intermediate powers such as displayed in trace $D$ in Fig. 2. Here ILSMs are formed during the early part of the pulse while the population in the degenerate modes is still small, and they remain isolated in frequency space above the dispersion curve after the degenerate mode population becomes large so both features are seen.

In this series of microwave experiments the instability that appears, when the lowest lying AFMR of $\mathrm{C}(2) \mathrm{CuCl}_{4}$ is driven to larger amplitudes, has been used to generate nonlinear excitations which are localized on a nano-length scale. To distinguish between the ILSMs of classical simulations and the excitations observed in experiment the latter will be identified as "anons." The hallmark experimental feature of the uniform mode breakup into anons is a broad and asymmetric spectral band below the AFMR. Classical MD simulations on a long 1-D antiferromagnetic spin chain show that both the amplitude of the extended spin wave at which it becomes unstable to break up into localized modes and the resulting spectral shape of the ILSMs are in good agreement with the microwave results. At still higher experimental powers the AFMR is observed to become stable again, a feature related to the fact that the pump pulse is longer than $T_{2}$.

Simulations have played an important role in identifying the most economical experimental pathway for the detection of anons in real solids. This interplay may be expected to continue since additional MD studies on a $\mathrm{C}(2) \mathrm{CuCl}_{4}$ spin chain in the presence of a magnetic field gradient indicate directed anon transport should be possible.

The authors thank H. Padamsee who provided the microwave amplifier for these experiments and also R. Lai and R.H. Silsbee for helpful discussions. This work is supported by NSF-DMR-9631298. One of the authors (U.T.S.) is supported in part by the Alexander von Humboldt-Foundation.

[1] P. M. Chaikin and T.C. Lubensky, Principles of Condensed Matter Physics (Cambridge University Press, Cambridge, England, 1995), Chap. 10.

[2] A. M. Kosevich, B. A. Ivanov, and A.S. Kovalev, Phys. Rep. 194, 117 (1990).

[3] H.-J. Mikeska and M. Steiner, Adv. Phys. 40, 191 (1991).

[4] A.J. Sievers and J.B. Page, in Dynamical Properties of Solids, edited by G. K. Horton and A. A. Maradudin (North-Holland, Amsterdam, 1995), Vol. 7, p. 137.

[5] S. Aubry, Physica (Amsterdam) 103D, 201 (1997).

[6] S. Flach and C. R. Willis, Phys. Rep. 295, 181 (1998).

[7] V.M. Burlakov, S. A. Kiselev, and V. N. Pyrkov, Phys. Rev. B 42, 4921 (1990).

[8] T. Dauxois and M. Peyrard, Phys. Rev. Lett. 70, 3935 (1993).

[9] S. Rakhmanova and D. L. Mills, Phys. Rev. B 58, 11458 (1998).

[10] F. M. Russell, Y. Zolotaryuk, and J. C. Eilbeck, Phys. Rev. B 55, 6304 (1997).

[11] P. Marquié, J. M. Bilbault, and M. Remoissenet, Phys. Rev. E 51, 6127 (1995).

[12] W.Z. Wang, J.T. Gammel, A.R. Bishop, and M.I. Salkola, Phys. Rev. Lett. 76, 3598 (1996).

[13] A. J. Sievers and S. Takeno, Phys. Rev. Lett. 61, 970 (1988).

[14] F. M. Russell and D. R. Collins, Nucl. Instrum. Methods Phys. Res., Sect. B 105, 30 (1995).

[15] B. I. Swanson et al., Phys. Rev. Lett. 82, 3288 (1999).

[16] R. Lai and A. J. Sievers, Phys. Rev. B 57, 3433 (1998).

[17] R. Lai and A. J. Sievers, Phys. Rev. Lett. 81, 1937 (1998).

[18] L.J. De Jongh, W. D. van Amstel, and A. R. Miedema, Physica (Utrecht) 58, 277 (1972).

[19] H. Reimann, H. Hagen, F. Waldner, W. Berlinger, and A. Arend, Solid State Commun. 17, 1319 (1975).

[20] R. Kubo and K. Tomita, J. Phys. Soc. Jpn. 9, 888 (1954). 